\title{
Evaluation of Mesh-under and Route-over Routing Strategies in AMI systems
}

\author{
Diego Fernando Ramírez Hincapie and Sandra Céspedes \\ Dpto. Tecnologías de Información y Comunicaciones \\ Universidad Icesi \\ Calle 18 No. 122-135, Cali, Colombia \\ diego.ramirez1@correo.icesi.edu.co and Sandra Céspedes
}

\begin{abstract}
The introduction of smart electric networks is fundamentally based on the integration of the electric grid with an intelligent and bidirectional communications network. As part of such communications network, the metering infrastructure is nowadays being automatized, so that the exchange of traffic between utilities and consumers happens in an efficient way. In this paper, we focus on the problem of routing in advance metering infrastructure (AMI) networks, and perform a qualitative comparison between the mesh-under and route-over routing strategies within the scope of AMI. The specific routing requirements for $A M I$ are identified, and an evaluation of the implications of both routing strategies on those requirements is performed, taking into account issues such as: scalability, endto-end delay, packet fragmentation, and retransmissions.
\end{abstract}

Index Terms-AMI, Mesh-under, Route-over, Routing, Smart grid communications, 6LoWPAN

\section{INTRODUCCIÓN}

Una de las principales metas que se tienen dentro de las nuevas iniciativas de las Tecnologías de Información y las Telecomunicaciones, en el contexto de las redes eléctricas, es la dotación de inteligencia a este tipo de infraestructuras. De esta manera se logra la implementación de nuevos servicios, que no solamente robustecen los mecanismos de generación y transporte de la energía, sino que también buscan construir un concepto de interactividad con el consumidor (bien sea residencial o corporativo), acercándose al esquema de flujo eléctrico y de datos bidireccional que se plantea en la llamada nueva red eléctrica inteligente, o Smart Grid.

Dentro del concepto de Smart Grid se propone que los usuarios residenciales puedan también generar energía por medio de fuentes renovables, la cual puede ser utilizada de manera local, y el exceso de energía que no sea consumido puede ser vendido al proveedor (o Utilidad) para su reutilización. Esta renovación apunta entonces a una red eléctrica con capacidad de toma de decisiones, amigable con el ambiente, proveedora de servicios interactivos con el consumidor (ya no es solamente el consumo lo que constituye el payload de la red en la vía Utilidad - Consumidor), y

(c) 2012 IEEE. Personal use of this material is permitted. Permission from IEEE must be obtained for all other uses, in any current or future media, including reprinting/republishing this material for advertising or promotional purposes, creating new collective works, for resale or redistribution to servers or lists, or reuse of any copyrighted component of this work in other works.

Published in Proc. COLCOM'12. DOI:10.1109/ColComCon.2012.6233670 finalmente, que se adapte a las necesidades y modelos de negocio del siglo XXI [1].

En el proceso de dicha evolución, millones de medidores inteligentes son utilizados como parte de una Infrastructura Avanzada de Medidores (AMI, por sus siglas en inglés), no solo para realizar lecturas del consumo del cliente y para enviar esta información a la Utilidad, si no también para habilitar el intercambio de otro tipo de información, e.g., precios en tiempo real. A futuro se espera que se incorporen un mayor número de dispositivos a la red AMI, particularmente displays y dispositivos HAN (Home Area Network), dedicados a la gestión energética (e.g., para balanceo de carga y autorregulación del consumo). Esto ha creado la necesidad de estudiar las tecnologías y protocolos de comunicación necesarios para habilitar el intercambio de información en AMI, teniendo en cuenta los requerimientos específicos y características particulares de estas redes, en cuanto a tipo de tráfico, condiciones técnicas de la red, número de medidores y escalabilidad, entre otras.

AMI tiene entonces dos características bien definidas: ha de ser fácil de implementar y debe proveer comunicación robusta. Por lo tanto, una de las tecnologías más promisorias para redes AMI ha sido las redes de sensores, que se comunican mediante tecnología inalámbrica 802.15.4. Las redes de sensores inalámbricas (WSNs, por sus siglas en inglés), cuya integración a los entornos residenciales y corporativos es cada vez mayor, se convierten en una buena alternativa para la interconexión de los contadores inteligentes en AMI, dado que proporcionan comunicación con buena cobertura y capacidades de control a bajo costo. Además, al formar Neighborhood Area Networks (NANs) inalámbricas, los costos de despliegue son bajos y la escalabilidad alta. Sin embargo, al combinar las WSN con el entorno geográfico, tamaño, y posibles aplicaciones a desplegar a futuro en las redes AMI, se crean nuevos retos en términos de los protocolos de comunicación necesarios para soportar estas redes.

Este artículo discute dichos retos y describe los protocolos necesarios para habilitar el intercambio de paquetes IP en una red AMI. En particular, se hace un estudio sobre los requerimientos específicos desde el punto de vista de enrutamiento. Dos estrategias de enrutamiento son evaluadas y comparadas: Mesh-under y Route-over, con el objetivo de analizar en 


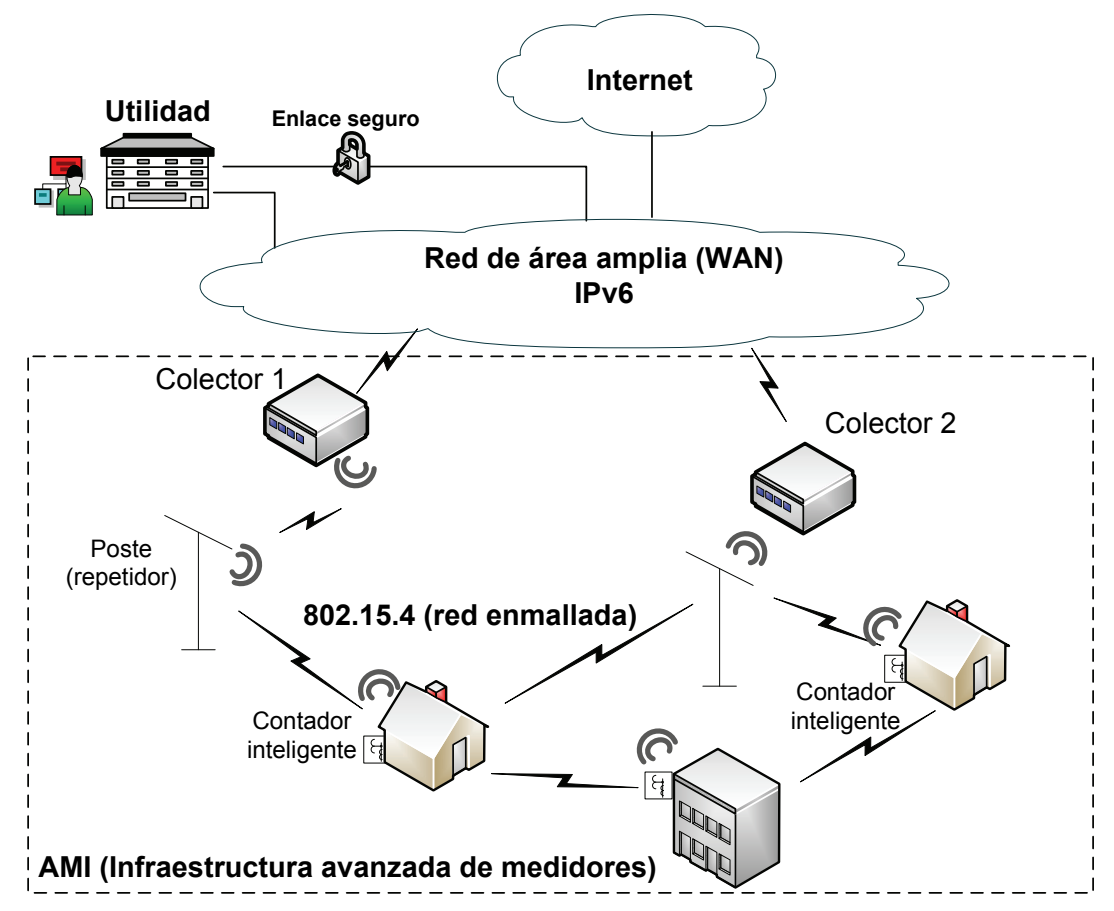

Fig. 1. Sistema de referencia: red AMI conectada mediante red de sensores inalámbricos 802.15.4

cuál nivel de la pila de protocolos convendría más tomar las decisiones de enrutamiento. El resto del artículo se encuentra organizado de la siguiente manera: en la Sección II se discute el sistema de referencia y las tecnologías y protocolos de WSN que se emplean en AMI, incluyendo la definición de las dos estrategias de enrutamiento en evaluación: mesh-under y route-over. En la Sección III discutimos los requerimientos de enrutamiento para redes AMI. En la Sección IV se realiza la comparación cualitativa de mesh-under y route-over cuando son empleadas en entornos AMI. Finalmente, las conclusiones y futuro trabajo son descritos en la Sección V.

\section{SISTEMA DE REFERENCIA}

La primera aproximación a una implementación de red inteligente es la instalación y gestión de una red avanzada de contadores AMI. Esta red se define como un sistema de comunicación bidireccional, que involucra nuevos servicios adicionales tanto para la Utilidad como para el consumidor. De un lado, el cliente tiene la posibilidad de conocer, en todo momento, sus patrones de consumo y consultar el precio de la electricidad, al tiempo que puede ahora participar activamente en el mercado de la industria eléctrica, al convertirse al mismo tiempo en proveedor. De otro lado, la Utilidad tendrá mayor capacidad de respuesta ante problemas súbitos o ante el aumento de consumo en periodos de alto costo (en cuyo caso bien podría enviarse una señal a los electrodomésticos inteligentes, para alertar sobre esta situación).

El sistema está formado por las siguiente redes: Home Area Networks (HANs), generalmente implementadas del lado del cliente; Neighborhood Area Networks (NANs), utilizadas para propósitos de recolección de información; y finalmente redes de área extendida (WANs), del lado de la Utilidad, de naturaleza pública o privada, y generalmente implementadas sobre Wi-Fi, WiMAX, fibra o redes celulares [2]. En el caso particular de las NANs y la interconexón de los contadores inteligentes, diferentes tecnologías han sido consideradas (e.g., mediante mensajes de texto en la red celular [SMS], o usando la misma red eléctrica como medio de transmisión con tecnología PLC [Power Line Communications]) [3]. Sin embargo, este estudio se enfoca en redes AMI interconectadas de forma inalámbrica, mediante tecnología inalámbrica 802.15.4 [4].

El modelo de la red en cuestión es ilustrado en la Fig. 1. En dicha red, cada contador cuenta con un radio 802.15.4, de manera que se crean conexiones ad hoc entre los nodos - formando una red en malla- y el tráfico fluye en ambos sentidos entre la Utilidad y los medidores [5]. Si bien la red de sensores inalámbrica puede estar basada en varias pilas de protocolos, para efectos del presente trabajo se ha adoptado la pila propuesta por el estándar IEEE 802.15.4 para las capas física y de nivel de enlace, derivando así en una LoWPAN. Adicionalmente, se considera que los paquetes de datos son IP, de manera que la red esté habilitada para interconectarse con otras redes IP (e.g., Internet), y ésta provea soporte a aplicaciones estándar desarrolladas sobre la pila de protocolos TCP/IP.

\section{A. IETF 6LoWPAN}

Dado que la red debe soportar el envío de paquetes IP, nuestro sistema también adopta el protocolo 6LoWPAN. El Grupo Especial sobre Ingeniería de Internet (IETF, por sus siglas en inglés) ha definido el estándar 6LoWPAN como alternativa para habilitar la transmisión de paquetes IPv6 sobre 
redes basadas en IEEE 802.15.4 (RFC 4944 [6]). Básicamente esta norma define una capa de adaptación, por debajo del nivel de red, que permite la adecuación de paquetes IPv6 (de tamaño mucho mayor al máximo permitido en 802.15.4) sobre la capa MAC del estándar IEEE 802.15.4. [7].

El enrutamiento en 6LoWPAN es un tema que reviste importancia en la medida en que las redes de sensores inalámbricas tienen limitaciones en potencia y capacidad de procesamiento de los nodos, tasa de transmisión, entre otros. Así, transmitir paquetes IPv6 sobre enlaces IEEE 802.15.4 no es una tarea trivial y supone varios desafíos importantes. De un lado están las restricciones que en sí mismas tienen los dispositivos de este tipo de redes, en términos de baja capacidad de procesamiento, baja capacidad de memoria y poco ancho de banda. Aunado a esto, la adopción de IPv6 como protocolo del nivel de red conlleva a una inconsistencia de especificaciones en MTUs (unidades máxima de transferencia): IPv6 requiere una MTU de al menos 1280 bytes, en tanto que la requerida por IEEE 802.15.4 es de un tamaño 10 veces menor. Adicionalmente, los encabezados de IPv6 aportan una alta sobrecarga (overhead) a la red, toda vez que tienen una longitud de 40 bytes, que sumados con los 8 bytes de nivel de transporte (UDP), los 25 bytes del encabezado MAC y los 21 bytes para seguridad del nivel de enlace, dejan disponible sólo 33 bytes para el payload [8].

En aras de hacer frente a estos problemas de requerimientos de MTU y reducción de overhead, IETF 6LoWPAN provee mecanismos para la fragmentación de paquetes, la compresión de encabezados IP y la reconstrucción de los mismos. En el caso que la tarea de enrutamiento se haga efectiva en esta capa 6LoWPAN, se estaría enrutando bajo la modalidad meshunder. De realizarse el enrutamiento en el nivel tradicional de red, se habla de una estrategia de enrutamiento route-over. Los detalles de ambas estrategias son discutidos a continuación.

\section{B. Enrutamiento Mesh-under}

Bajo esta estrategia no se realiza ningún tipo de enrutamiento a nivel de red. La capa de adaptación direcciona los paquetes al destino en múltiples saltos de radio, es decir, el enrutamiento se hace sobre el nivel de enlace basado en una trama tipo 802.15.4 o en un encabezado 6LoWPAN [9]. En caso de ser necesario, el paquete IP a ser enrutado es fragmentado por la capa de adaptación y luego los fragmentos son entregados al siguiente hop, hasta que eventualmente alcanzan el destino. Los fragmentos pueden viajar a través de diferentes caminos y, una vez son enrutados exitosamente, la capa de adaptación del destino crea el paquete IP correspondiente.

En aras de lograr el reenvío de paquetes por múltiples saltos (multi-hop), IETF 6LoWPAN ha definido un encabezado llamado "Mesh-Header", que tiene como función principal codificar el límite de saltos (número de segmentos de red al que el paquete puede ser reenviado antes de ser descartado por el enrutador), así como la fuente y el destino de los paquetes en el nivel de enlace. Las direcciones empleadas en el MeshHeader pueden ser de 16 o 64 bits, de acuerdo a lo indicado por el estándar 802.15.4. La cantidad máxima de saltos se ha especificado por un total de 255 , cuyo valor es utilizado en el campo de saltos faltantes y disminuido por cada hop que reenvía el paquete. Los paquetes de descartarán entonces cuando el valor en el campo de saltos faltantes llegue a cero y no se haya alcanzado el destino final de dicho paquete [8].

\section{Enrutamiento Route-over}

En esta estrategia de enrutamiento las decisiones se toman en el nivel de red, de tal manera que cada nodo funciona como un enrutador IP. De acuerdo a esto, el direccionamiento de paquetes utiliza las tablas tradicionales de enrutamiento IP y la opción de salto por salto de IPv6 que está incluida en el encabezado IP. Para este efecto, la capa de adaptación de 6LoWPAN establece un mapeo directo entre la trama y los encabezados IP [7]. La tabla de enrutamiento se utiliza en el momento en que se definen los saltos a los que se enviarán los fragmentos de los paquetes a enrutar. La capa de adaptación de IPv6 verifica que los fragmentos han llegado a su destino exitosamente, crea el paquete IP correspondiente y se lo entrega a la capa superior —el nivel de red- que finalmente direcciona o efectúa algún proceso sobre el paquete basado en la información provista por la tabla de enrutamiento.

\section{ReQuerimientos DE ENRUTAMIENTo EN AMI}

Las redes AMI que emplean WSN para conectar sus nodos son consideradas un subconjuto de lo que se ha denominado redes de baja potencia con pérdidas (Low-power and Lossy Networks, o LLN por sus siglas en inglés). Esto debido a las condiciones geográficas en las que se despliegan las redes AMI, en donde las conexiones contador a contador sufren de interferencia y pérdidas debido a obstáculos temporales (e.g., vehículos que obstruyen la línea de vista), o a condiciones adversas (e.g., despliegues en zonas rurales). Dada la alta inestabilidad y variabilidad en la calidad de los enlaces, se hace necesario contar con protocolos robustos que garanticen la entrega oportuna y eficaz de la información.

Dentro del conjunto de requerimientos de enrutamiento especificados para LLNs, y de acuerdo a las características de las redes AMI descritas en la Sección II, se han definido los siguientes requerimientos que han de ser satisfechos por las estrategias de enrutamiento a implementar en estos sistemas [10]:

1) Escalabilidad: El protocolo debe ser capaz de soportar la organización de un gran número de nodos (en un rango entre 100 y 10000). De esta manera, el protocolo debe garantizar la escalabilidad necesaria para acomodar un número de nodos grande y creciente, sin deteriorar el desempeño de la red. Por otra parte, los protocolos de enrutamiento a implementar deben ser capaces de soportar la organización de tal número de nodos en regiones de tamaño configurable.

2) Restricción de recursos y/o parámetros: El protocolo debe soportar enrutamiento con recursos limitados (como tamaño de memoria, CPU, nivel de batería, etc). 
Es decir, el protocolo ha de ser capaz de advertir las capacidades del nodo que van a ser requeridas para la decisión de enrutamiento. El enrutamiento dentro de la red AMI debería requerir que los nodos que la forman tengan la capacidad de computar dinámicamente, definir y seleccionar diferentes rutas hacia el mismo destino, dependiendo de la naturaleza del tráfico. Esto implicaría el uso de otros mecanismos para marcar o etiquetar el tráfico para la decisión apropiada de enrutamiento usando el formato del paquete IPv6.

3) Funcionamiento dinámico: El protocolo debería ser capaz de adaptarse dinámicamente a través de la aplicación de métricas de enrutamiento apropiadas ante condiciones cambiantes en la comunicación, por ejemplo, degradación de la calidad de los enlaces, naturaleza variable del tráfico, o movilidad de los nodos. El protocolo debe ser capaz además de computar, seleccionar y optimizar dinámicamente los múltiples caminos que serán usados por los dispositivos participantes para direccionar el tráfico.

4) Soporte a alto número de flujos de tráfico: El protocolo debería ser capaz de utilizar un gran número de flujos de tráfico para facilitar los requerimientos 1 y 2 .

5) Interoperabilidad: El protocolo debería soportar internetworking cuando se conecte a redes que usan protocolos idénticos. Adicionalmente, la red debe tener la habilidad para interactuar con otras redes, mediante mecanismos como redistribución de rutas, cuando las otras redes empleen protocolos diferentes.

6) Adaptación al cambio: Si bien la movilidad es baja en las LLNs - y en particular para el caso de AMIel dinamismo de la red debido a la asociación de los nodos ha de ser un tema a considerar. En este sentido, la red local (residencial o corporativa, según la naturaleza del cliente) no debería impactar la red entera en caso de necesitar ser reconfigurada. El protocolo debería soportar mecanismos apropiados que le permitan enterarse de cambios en la red y en la conectividad. El protocolo debería usar esta información para iniciar los procedimientos necesarios para la reorganización y/o reconfiguración, y mantener así la eficiencia del enrutamiento.

7) Latencia: Si bien las LLNs son tolerantes al retardo, en el caso particular de AMI el protocolo de enrutamiento implementado en la red debería ser capaz de soportar la habilidad para enrutar de acuerdo a diferentes métricas (entre ellas, el retardo).

8) Seguridad: Este aspecto reviste especial importancia en las redes AMI, toda vez que se requiere protección de la información que viaja en ambas vías, entre cliente y proveedor del servicio. Indicadores o patrones de consumo del usuario, alertas por indisponibilidad o corte provisional del servicio, así como datos de facturación, constituyen información sensible que debe ser salvaguardada.
En la siguiente sección se realiza una evaluación de las estrategias de enrutamiento frente a los requerimientos en entornos AMI previamente descritos.

\section{Mesh-Under VS. ROUTE-OVER}

Es posible evaluar el desempeño de los protocolos de enrutamiento en entornos de redes AMI en términos de diferentes parámetros: entrega de paquetes, varianza en el retardo de paquetes (PDV, por sus siglas en inglés), escalabilidad de la red, número de hops soportados, y la habilidad de manejar densidades variables en la red. La escalabilidad se constituye en una variable importante a considerar, toda vez que a mayor tamaño de la red, mayor es la probabilidad de su congestión; esto a su vez genera un mayor delay y valores más altos de PDV, lo que al final resulta en un desempeño menor. Por otra parte, aun cuando se trata de una red estática, las condiciones de las redes AMI (i.e., entorno en las que son desplegadas, distancias y obstáculos del entorno), hacen que la trasmisión de paquetes en estas redes no sea una tarea trivial.

A continuación, más que evaluar protocolos de enrutamiento específicos para redes AMI, se evalúan mesh-under y routeover como estrategias de enrutamiento (dentro de las cuales se pueden clasificar los protocolos de enrutamiento para AMI). De esta forma, se contribuye en la identificación de la capa en la cual se hace más conveniente tomar las decisiones de enrutamiento, considerando las características particulares de este tipo de redes.

De acuerdo a las características de las estrategias de enrutamiento propuestas para 6LoWPAN, se pueden identificar dos aspectos de especial importancia que suscitan el debate entre estos esquemas: 1) las implicaciones que tiene el enrutamiento a nivel de enlace; y 2) la decisión sobre la capa en la que tiene lugar el enrutamiento de datagramas dentro de la LoWPAN. Como se mencionó en la Sección II, bajo una modalidad mesh-under, el enrutamiento toma lugar en la capa de adaptación, y esto se logra a partir de la emulación de un dominio de broadcast único en un solo enlace lógico [11]. Por otro lado, route-over ejecuta el enrutamiento a nivel de red, donde cada nodo actúa como enrutador IP, difiriendo así de la modalidad mesh-under, al tener múltiples dominios de broadcast asociados a cada uno de los enlaces de radio existentes dentro de la LoWPAN, lo que permite una mayor integración de tecnologías de enlace.

En cuanto a la transmisión efectiva de la información en una red AMI con alta densidad de nodos, es decir su escalabilidad y efectividad en la entrega de paquetes, se observa que bajo la modalidad de mesh-under, en caso de pérdida de uno o más fragmentos en un salto intermedio, todos los fragmentos deben ser retransmitidos desde la fuente al destino. Es decir que, ante un escenario de pérdida de fragmentos en saltos intermedios en mesh-under, se genera un "desgaste" de energía innecesario toda vez que se propagan fragmentos de manera independiente hacia los otros saltos, aun cuando el paquete no podrá ya reensamblarse en el destino final.

Por otra parte, en el caso de route-over, la retransmisión no ocurre desde la fuente hasta el destino, sino desde el hop 
TABLA I

PARÁmETRos DE EVALUACIÓN

\begin{tabular}{|l|l|l|}
\hline Parámetro & Descripción & Valor \\
\hline$T_{w}$ & Retardo debido al período de contención & $5 \mu \mathrm{s}$ \\
\hline$T_{p}$ & Retardo de propagación del enlace & $2.5 \mathrm{~ms}$ \\
\hline$h$ & Número de saltos & $1 \sim 150$ \\
\hline$f$ & Número de fragmentos del paquete IP & 1 \\
\hline$T_{n p}$ & Tiempo de procesamiento por nodo & $100 \mu \mathrm{s}$ \\
\hline$\delta$ & Tiempo de re-ensamble de fragmentos & $100 \mu \mathrm{s}$ \\
\hline$p$ & Probabilidad de llegada salto-a-salto & $50 \% \sim 80 \%$ \\
\hline$N$ & Reintentos de entrega salto-a-salto de un fragmento & 4 \\
\hline
\end{tabular}

en que se perdieron los fragmentos hasta el siguiente salto, mediante un mecanismo de almacenamiento en buffer del siguiente hop. Así, una vez que se reciben todos los fragmentos que previamente se habían perdido, la capa de adaptación reconstruye el paquete IP original. Route-over proporciona entonces una recuperación de paquetes salto a salto pues, a diferencia de mesh-under, cada fragmento no es tratado de manera independiente. Sin embargo, dado que los nodos que forman la red AMI son dispositivos limitados en potencia y capacidad de procesamiento, el almacenamiento de los fragmentos en el buffer supone la limitante de su tamaño. En el caso de que la red presente congestión de tráfico, los nodos intermedios son susceptibles a presentar un desbordamiento del buffer, situación que no tendría efectos adversos en la presencia de una implementación mesh-under, dado que no existe una recuperación de la información perdida salto por salto [9].

Debido a que cada estrategia presenta ventajas y desventajas, a continuación se argumentan las razones para utilizar una o la otra, dependiendo del tipo de aplicación y las características de la red AMI en la que se despliegan. En el caso particular de redes AMI con una alta densidad de nodos, y con altas tasas de tráfico pero con tamaños de paquetes pequeños (i.e., paquetes que no requieren fragmentación), resultaría conveniente el enrutamiento mesh-under, ya que bajo un esquema route-over existe posibilidad de desbordamiento de buffer en nodos intermedios. Adicionalmente, al no haber fragmentación, el tiempo y recursos en procesamiento se reducen, ya que el paquete se mueve salto a salto sin tener que procesar los encabezados IP en los nodos intermedios.

Este comportamiento es ilustrado en la Fig. 2, en donde para el sistema de referencia descrito en la Sección II (Fig. 1) se calcula el retardo total para dos flujos IP, uno de 50 paquetes y otro de 500 paquetes en total. Para obtener los datos comparativos en Matlab, se han empleado los parámetros y valores especificados en la Tabla I. El retardo salto a salto es calculado de acuerdo a Chowdhury et al. [9]:

$$
\begin{aligned}
R_{\mathrm{MU}} & =\left(T_{w}+T_{p}\right) h+T_{n p}(h-1)+(f-1)\left(T_{w}+T_{p}\right) \\
R_{\mathrm{RO}} & =\left(T_{w}+T_{p}\right) f \times h+(h-1)\left(T_{n p}+\delta\right)
\end{aligned}
$$

donde $R_{\mathrm{MU}}$ y $R_{\mathrm{RO}}$ especifican el retardo end-to-end en meshunder y route-over respectivamente.

Por otro lado, cuando existe fragmentación de paquetes debido a aplicaciones que envían altas cantidades de información, y cuando la calidad de los enlaces en la red AMI es altamente variable, el requerimiento de mayor relevancia a cubrir pasa a ser la integridad y confiabilidad de la red. En dicha situación el esquema route-over puede proporcionar un mejor desempeño, debido al mecanismo de recuperación salto por salto que implementa, y que resulta más robusto que aquel utilizado por mesh-under. Adicionalmente, cuando se consideran la adaptación al cambio y el dinamismo que se exige de las redes AMI, éstos tienen una mayor probabilidad de ser alcanzadas bajo un esquema route-over en el escenario descrito, dada la confiabilidad y robustez que proporciona esta estrategia de enrutamiento.

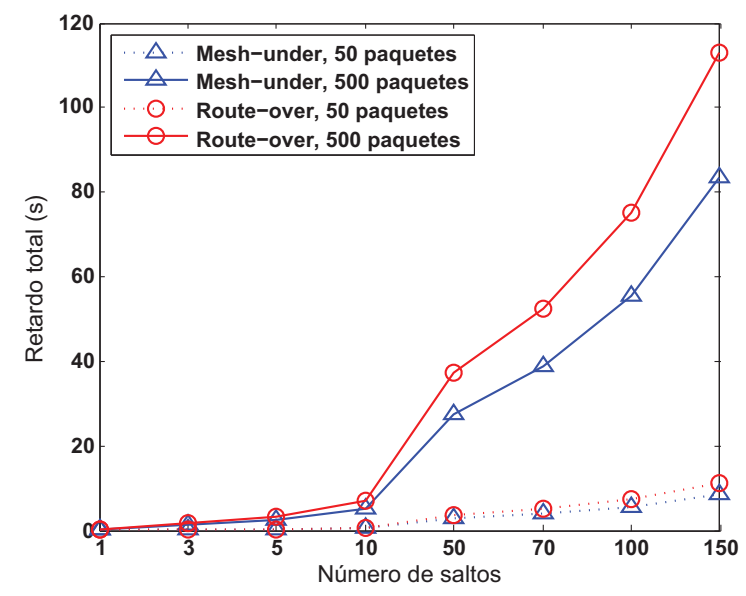

Fig. 2. Retardo total para diferentes flujos IP sin fragmentación de paquetes.

Este comportamiento es ilustrado en la Fig. 3, en donde se calcula la probabilidad de llegada para un fragmento IP, 
en redes con pérdida (i.e., baja probabilidad de llegada saltoa-salto). No obstante, es importante resaltar que la robustez obtenida para route-over viene acompañada de un mayor retardo en la entrega de paquetes. La probabilidad salto a salto es calculada de acuerdo a las siguientes ecuaciones [9]:

$$
\begin{aligned}
P_{\mathrm{MU}} & =\left(\sum_{i=1}^{N} p(1-p)^{i-1}\right)^{h} \\
P_{\mathrm{RO}} & =\sum_{i=1}^{N} p(1-p)^{i-1}
\end{aligned}
$$

donde $P_{\mathrm{MU}}$ y $P_{\mathrm{RO}}$ indican la probabilidad de llegada end-toend de un fragmento IP en mesh-under y route-over respectivamente.

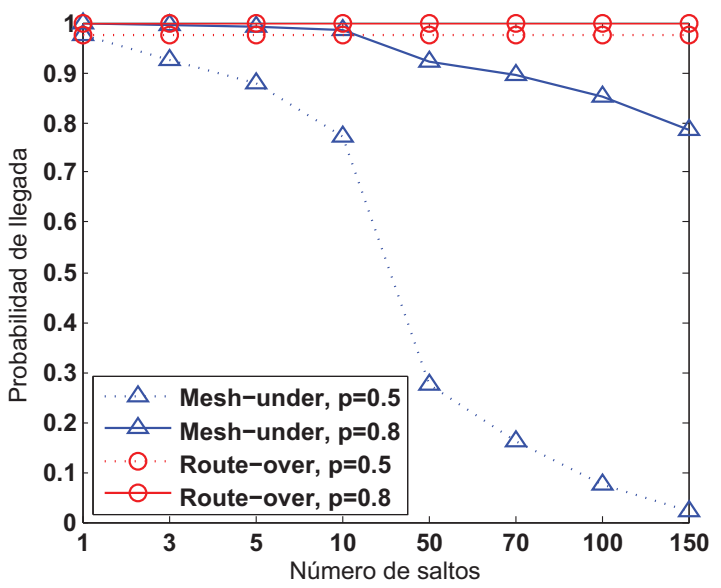

Fig. 3. Probabilidad de llegada end-to-end de un fragmento IP sobre diferentes números de saltos en redes AMI con pérdidas.

\section{Conclusiones}

En este artículo se ha presentado una evaluación comparativa entre las estrategias de enrutamiento propuestas para 6LoWPAN, en el contexto de su aplicación en redes de contadores inteligentes AMI. Se concluye que los requerimientos de enrutamiento para redes AMI pueden ser obtenidos a partir de aquellos definidos para redes de baja potencia y con pérdidas (Low-power and Lossy Networks), y que los esquemas de enrutamiento mesh-under y route-over hacen frente a estos requerimientos en menor o mayor medida, según el escenario de estudio. Un requerimiento importante dentro de AMI es la escalabilidad de la red, en cuyo caso un esquema route-over proporciona mejor desempeño que aquel provisto por mesh-under para los casos en que la fragmentación de paquetes IP es necesaria. El caso contrario ocurre si el requerimiento a cubrir es el de un menor retardo en la entrega de paquetes, especialmente cuando el tamaño de dichos paquetes es pequeño y no requieren fragmentación. En este caso, un esquema mesh-under resulta ser mejor opción de implementación, dado que no se efectúa un mecanismo de verificación de entrega y recuperación de paquetes tan robusto como el provisto por route-over. En el futuro se espera validar cuantitativamente las observaciones realizadas, a través de simulaciones en la herramienta OMNET++ de protocolos de enrutamiento representativos de cada estrategia.

\section{REFERENCIAS}

[1] C. A. Díaz Andrade and J. C. Hernández, "Smart Grid: Las TICs y la modernización de las redes de energía eléctrica - Estado del Arte," Sistemas \& Telemática, vol. 9, no. 18, pp. 53-81, 2011.

[2] R. Acra and M. Thaker, "Internet Standards Come to the Advanced Metering Infrastructure," Electric Energy Publications Inc., 2011. [Online]. Available: http://www.electricenergyonline.com/?page=show_ article \&mag $=66 \&$ article $=525$

[3] T. Khalifa, K. Naik, and A. Nayak, "A Survey of Communication Protocols for Automatic Meter Reading Applications," IEEE Communications Surveys \& Tutorials, vol. 13, no. 2, pp. 168-182, 2011.

[4] IEEE 802.15WPAN Task Group 4g (TG4g) Smart Utility Networks, "IEEE 802.15 Smart Utility Networks (SUN) amendment to 802.15.4."

[5] S. Cespedes, A. A. Cardenas, and T. Iwao, "Comparison of data forwarding mechanisms for ami networks," in Proc. of IEEE PES Innovative Smart Grid Technologies (ISGT), 2012, pp. $1-8$.

[6] G. Montenegro, N. Kushalnagar, J. Hui, and D. Culler, "Transmission of IPv6 Packets over IEEE 802.15.4 Networks," IETF Secretariat, RFC 4944, 2007.

[7] N. Kushalnagaret, G. Montenegro, and C. Schumacher, "IPv6 over Low-Power WirelessPersonal Area Networks (6LoWPANs): Overview, Assumptions, Problem Statement, and Goals," IETF Secretariat, RFC 4919, 2007.

[8] G. Ee, C. Ng, N. Noordin, and B. Ali, "A Review of 6LoWPAN Routing Protocols," in Proc. of Asia-Pacific Advanced Network (APAN), 2011.

[9] A. H. Chowdhury, M. Ikram, H.-S. Cha, H. Redwan, S. M. S. Shams, K.-H. Kim, and S.-W. Yoo, "Route-over vs Mesh-under Routing in 6LoWPAN," in Proc. of the International Conference on Wireless Communications and Mobile Computing (IWCMC), 2009, pp. 12081212.

[10] M. Dohler, T. Watteyne, T. Winter, and D. Barthel, "Routing Requirements for Urban Low-Power and Lossy Networks," IETF Secretariat, RFC 5548, 2009.

[11] J. Hui and D. Culler, "Extending IP to Low-Power, Wireless Personal Area Networks," IEEE Internet Computing, vol. 12, no. 4, pp. 37-45, july-aug. 2008. 